\title{
NMR Study of Multiferroic Iron Niobate Perovskites
}

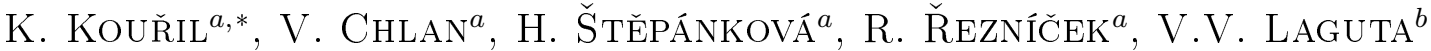 \\ AND I.P. RAEVSKI ${ }^{c}$ \\ ${ }^{a}$ Charles University in Prague, Faculty of Mathematics and Physics, \\ V Holešovičkách 2, 18000 Prague 8, Czech Republic \\ ${ }^{b}$ Institute of Physics AS CR, Cukrovarnická 10, 16200 Prague 6, Czech Republic \\ ${ }^{c}$ Institute of Physics, Southern Federal University, Rostov on Don 344090, Russia
}

\begin{abstract}
We present ${ }^{93} \mathrm{Nb},{ }^{207} \mathrm{~Pb}$ and ${ }^{135,137} \mathrm{Ba} \mathrm{NMR}$ study of multiferroic lead iron niobate and barium iron niobate ceramics. We ascribe development of NMR spectra on cooling below room temperature to magnetism of ferric ions close to resonating nuclei and introduce a framework capable of describing key features of the observed temperature induced changes. We show that the ferric ions have much stronger influence on hyperfine fields at nuclei of large cations in lead compound and estimate strength of interaction of superantiferromagnetic clusters.
\end{abstract}

DOI: 10.12693/APhysPolA.127.234

PACS: $76.60 .-\mathrm{k}, 76.60 . \mathrm{Jx}, 75.10 . \mathrm{Nr}, 75.85 .+\mathrm{t}$

\section{Introduction}

Lead iron niobate $\mathrm{PbFe}_{0.5} \mathrm{Nb}_{0.5} \mathrm{O}_{3}$ (PFN) has been subject of interest because of its magnetoelectric properties. It forms solid solution with barium iron niobate $\mathrm{BaFe}_{0.5} \mathrm{Nb}_{0.5} \mathrm{O}_{3}$ (BFN). Substitution of $\mathrm{Pb}$ by $\mathrm{Ba}$ has been reported to significantly alter magnetic properties of the material [1].

PFN and BFN are $\mathrm{ABO}_{3}$ compounds and form perovskite crystal structure, $\mathrm{Ba}$ and $\mathrm{Pb}$ ions occupy A sites, $\mathrm{Nb}$ and $\mathrm{Fe}$ share the $\mathrm{B}$ sites. The only magnetic ions in the compounds are the ferric ions; since they share the $\mathrm{B}$ sublattice with non-magnetic Nb the resulting disorder plays key role in magnetic properties [2].

Upon cooling the system undergoes several ferroelectric and magnetic phase transitions $[1,3,4]$. While the ferroelectric transitions happen above room temperature (RT) the magnetic ones take place well below it. PFN becomes partly antiferromagnetic (AFM) below $T_{\mathrm{N}}=147 \mathrm{~K}$ while remainder of material forms superantiferromagnetic clusters (SAF). Part of the SAF clusters freezes below temperature $T_{\mathrm{g}}=10 \mathrm{~K}$ thus forming a cluster glass (CG). In BFN only transition from paramagnetic to $\mathrm{CG}$ phase has been observed at temperature $T_{\mathrm{g}}=25 \mathrm{~K}[1]$.

In this paper we discuss temperature induced changes of ${ }^{93} \mathrm{Nb}$ and large cations $\left({ }^{207} \mathrm{~Pb}\right.$ and $\left.{ }^{135,137} \mathrm{Ba}\right) \mathrm{NMR}$ spectra in PFN and BFN. We propose that observed changes of spectral shapes can be understood in terms of simple model based on influence of nearest neighbor (nn) ferric ions on resonant nuclei.

\section{Experimental}

Ceramic samples of PFN and BFN were prepared by solid state reaction route. For technological reasons the

*corresponding author; e-mail: karel.kouril@mff.cuni.cz
PFN sample contained addition of lithium. Phase purity was checked by X-ray.

Frequency swept NMR spectra were measured in 9.41 T NMR cryomagnet (Larmor frequencies corresponding to magnet field are: $97.96 \mathrm{MHz}$ for ${ }^{93} \mathrm{Nb}$, 83.73 $\mathrm{MHz}$ for ${ }^{207} \mathrm{~Pb}, 44.48 \mathrm{MHz}$ for ${ }^{137} \mathrm{Ba}$ and $39.76 \mathrm{MHz}$ for ${ }^{135} \mathrm{Ba}$ ) using modified (Q)CPMG pulse sequence where all echoes are recorded. Well matched tunable probeheads were used in all experiments except for these at temperatures below $4.2 \mathrm{~K}$ which were performed using a non-tuned broadband probehead. Spectra are plotted either as an integral of Fourier-transformed echo taken in interval $\pm(0.1-0.3) \mathrm{MHz}$ around excitation frequency (ft-integral), or as envelope of Fouriertransformed echoes recorded at individual excitation frequencies (ft-envelope). In presented temperature dependences of ${ }^{93} \mathrm{Nb}$ and ${ }^{207} \mathrm{~Pb}$ spectra only the first echo was used for evaluation in order to show broad spectral component (see below), in ${ }^{135,137} \mathrm{Ba}$ spectra several tens of echoes were necessary to obtain good signal-tonoise ratio.

\section{Results and discussion}

${ }^{93} \mathrm{Nb}$ spectra (Fig. 1) can generally be seen as superposition of three components: broad, narrow and very narrow. Broad components have faster transversal relaxation than the narrow and very narrow ones. The very narrow component in BFN spectrum is more difficult to detect - one needs to exclude first recorded echo from evaluation in order to suppress the broad component and make the much weaker narrow and very narrow ones observable. At RT narrow and broad components are of similar linewidths. While width of the narrow components increases noticeably on cooling, much more substantial broadening is observed on the broad ones. On further cooling the broad components vanish below $60 \mathrm{~K}$ and $50 \mathrm{~K}$ and gradually reappear below $20 \mathrm{~K}$ and $5 \mathrm{~K}$ in BFN and PFN, respectively. This behavior appears to be governed by temperature dependence 
of transversal relaxation. Width of the broad components at lowest temperatures exceeds available tuning range and its center is significantly shifted from Larmor frequency corresponding to the applied magnetic field.

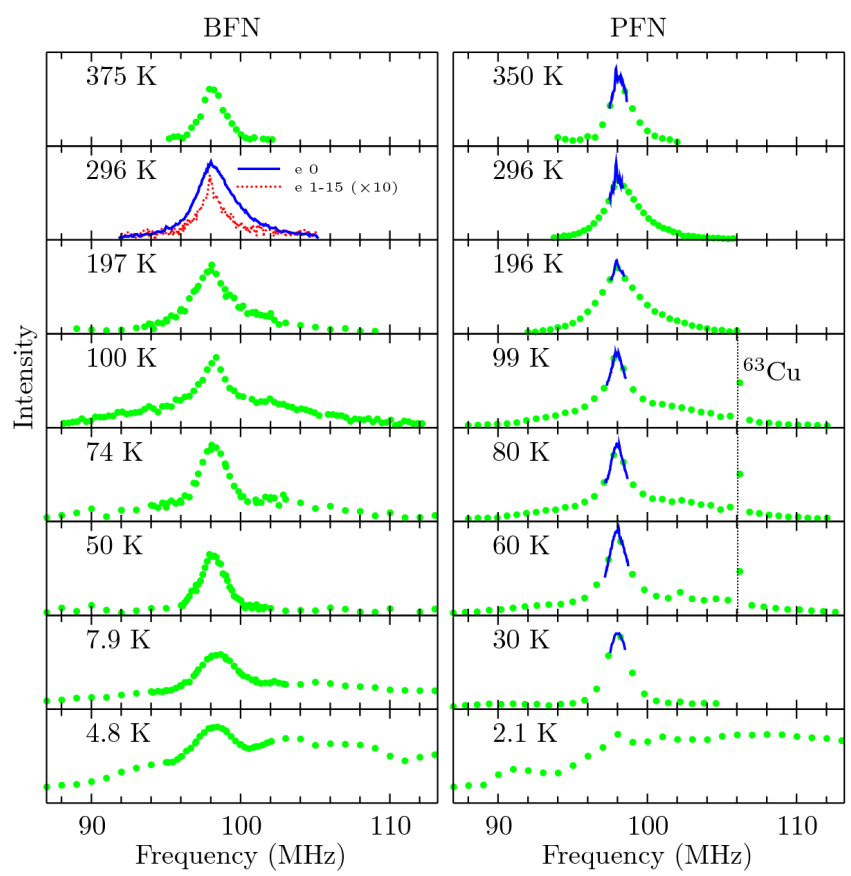

Fig. 1. ${ }^{93} \mathrm{Nb}$ NMR spectra of BFN (left) and PFN (right) at selected temperatures. Points correspond to $\mathrm{ft}$-integral evaluation, lines correspond to more detailed $\mathrm{ft}$-envelope. Two different evaluations are presented for $296 \mathrm{~K}$ spectrum of PFN, first uses only the first recorded echo and shows the broad component, second uses the remaining recorded echoes and shows the narrow and very narrow components (this spectrum is magnified by factor of 10). Spurious NMR signal originating in copper coil can be seen in PFN spectra.

In Fig. 2 there is ${ }^{93} \mathrm{Nb}$ spectrum of PFN at $3.1 \mathrm{~K}$ where whole broad component can be seen. In contrast to temperatures above $50 \mathrm{~K}$ has its center frequency shifted almost $20 \mathrm{MHz}$ above ${ }^{93} \mathrm{Nb}$ Larmor frequency and the shift is dependent on sequential number of echo used for evaluation.

Spectra of ${ }^{207} \mathrm{~Pb}$ in $\mathrm{PFN}$ and ${ }^{135,137} \mathrm{Ba}$ in $\mathrm{BFN}$ are shown in Fig. 3, in general these can be seen as combination of narrow and broad component as well. The ${ }^{207} \mathrm{~Pb}$ line shows monotonous broadening of both components upon cooling and whole line vanishes at temperatures below $30 \mathrm{~K}$. The ${ }^{135,137} \mathrm{Ba}$ lines in BFN broaden upon cooling as well, below $150 \mathrm{~K}$ broad components appear in the spectra.

Our ${ }^{93} \mathrm{Nb}$ data on PFN ceramics are in good agreement with the previously published results [5]. The very narrow component in PFN has been ascribed to central transition of ${ }^{93} \mathrm{Nb}$ in Fe-poor regions [5]. This component is weaker in BFN than in PFN, which indicates that in BFN the distribution of Fe is likely more homogeneous.

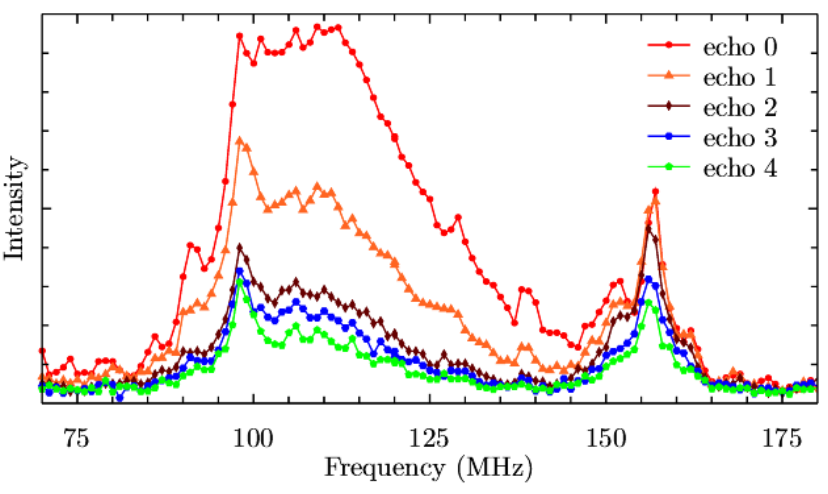

Fig. 2. Ft-integral ${ }^{93} \mathrm{Nb}$ NMR spectra of PFN at $3.1 \mathrm{~K}$ evaluated from different echoes in QCPMG pulse sequence (echo-to-echo separation was set to $40 \mu \mathrm{s}$ ) showing modulation of spectral shape by transversal relaxation. At $156 \mathrm{MHz}$ there is ${ }^{7} \mathrm{Li} \mathrm{NMR}$ signal.

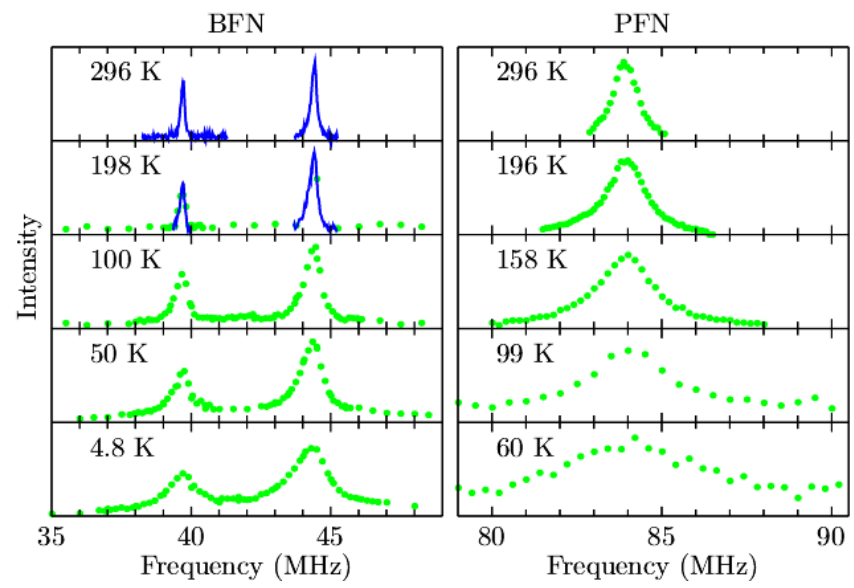

Fig. 3. ${ }^{135,137} \mathrm{Ba}$ and ${ }^{207} \mathrm{~Pb}$ spectra of $\mathrm{BFN}$ and $\mathrm{PFN}$ at selected temperatures. Points correspond to $\mathrm{ft}$ integral evaluation, lines correspond to more detailed ft-envelope.

General similarity of temperature behavior of BFN and PFN ${ }^{93} \mathrm{Nb}$ spectra implies similar sequence of magnetic phase transitions in both systems.

Width of observed resonance lines is given by two factors: electron magnetism of nearby ferric ions and electric quadrupole interaction of nucleus with its close surroundings (except ${ }^{207} \mathrm{~Pb}$ which is spin $1 / 2$ nucleus with no quadrupole moment). The quadrupole interaction is sensitive to structural transformations - its contribution should not change significantly unless configuration of $n n$ ions changes. Since the structural transformations take place above RT we consider the magnetism to be the responsible for the dramatic changes of spectra below RT.

It is clear from width of spectra that the additional fields due to electron magnetism are at order of few $\mathrm{T}$ - about an order of magnitude larger than can be expected for dipole fields. We propose that transferred hyperfine fields are responsible for presence of 
broad components in spectra of all non-magnetic ions. The transferred fields arise as follows: Electrons from valence $s$-shell of target non-magnetic ion are polarized by adjacent ferric ions by mechanism resembling superexchange interaction. The polarized $s$-electrons then create strong contact magnetic field on nucleus of the target ion. Contribution of each ferric ion to this field is parallel with its magnetic moment.

Following model can be applied to shed light on presence of narrow and broad components in observed spectra: Total transferred field on a particular nucleus is a superposition of transferred fields generated by individual $n$ n ferric ions in B sites (a non-magnetic ion in A or $B$ site has 8 or 6 nn B sites respectively, which can be occupied by the ferric ions). The individual contributions to the total transferred field can either cancel out, or give rise to a strong transferred field. To simplify discussion, we assume that magnetic moments of the nn ferric ions are collinear and that all $\mathrm{nn}$ ferric ions are equivalent in terms of strength of the transferred fields which they generate on nucleus of target non-magnetic ion. Under the assumptions, two distinct situations arise: either numbers of spin up and spin down ferric nn are equal, or they are not. In the first case the total transferred field vanishes and the ion contributes to the narrow component (which is only broadened by much weaker dipole fields). In the second case there is a significant transferred field on nucleus and the ion contributes to the broad component.

In paramagnetic region well above $T_{\mathrm{N}}$ rapid fluctuations of magnetic moments lead to low time-average of their contribution to local field on resonant nuclei and resonance lines are narrow. On cooling the fluctuations slow down, distributions of time-averaged dipole and transferred fields broaden, hence narrow as well as broad component of resonance lines broaden. Slowing of local fields fluctuations also increases efficiency of transversal relaxations which eventually leads to vanishing of broad components on ${ }^{93} \mathrm{Nb}$ spectra and of entire ${ }^{207} \mathrm{~Pb}$ spectrum. As temperature is lowered even further the fluctuations are gradually extinguished and the broad components reappear in spectra. There is no distinct line on frequency of ${ }^{207} \mathrm{~Pb}$ resonance in PFN at lowest temperatures - either the resonance line is too broad to be unambiguously identified or the transversal relaxations are still too fast at these temperatures.

We ascribe the broad component of PFN and BFN ${ }^{93} \mathrm{Nb}$ spectra at the lowest temperatures to frozen SAF clusters. Overall shift of the component to high frequencies shows that there is preferential orientation of magnetic moments of the clusters parallel with the applied field. Such preference indicates that inter-cluster interactions, responsible for freezing of SAF cluster glass, are of strength comparable to that of interaction of individual clusters with the applied magnetic field.
From large cation spectra it can be seen that ${ }^{207} \mathrm{~Pb}$ in $\mathrm{PFN}$ is influenced by ferric ions much more profoundly than ${ }^{135,137} \mathrm{Ba}$ in $\mathrm{BFN}$. Despite ${ }^{207} \mathrm{~Pb}$ being spin $1 / 2$ nucleus (hence sensitive solely to broadening by magnetic interactions), its resonance line is much broader than these of ${ }^{135,137} \mathrm{Ba}$ which at temperatures above $200 \mathrm{~K}$ are mainly broadened by quadrupole interaction (ratio of widths of ${ }^{135,137} \mathrm{Ba}$ lines approximately corresponds to ratio of quadrupole moments of the isotopes). In ${ }^{135,137}$ Ba spectra magnetic broadening becomes significant only at lower temperatures where broad components emerge and ratio of widths of the narrow components approaches that of gyromagnetic ratios of the nuclei. This shows that in PFN the local magnetic fields generated by $\mathrm{nn}$ ferric ions on ${ }^{207} \mathrm{~Pb}$ nuclei in $\mathrm{A}$ sites are much higher than in these fields on ${ }^{135,137} \mathrm{Ba}$ in $\mathrm{A}$ sites in BFN.

\section{Conclusions}

Presented NMR spectra can be interpreted in terms of effect of $n n$ ferric ions on resonating nuclei. The spectra indicate similar sequence of magnetic transitions in both materials on cooling. Local magnetic fields generated by ferric ions on nuclei in A sites are much weaker in BFN than in PFN. SAF clusters freeze preferentially with their magnetic moments parallel with applied magnetic field, thus providing upper-bound estimate of inter-cluster interaction strength.

\section{Acknowledgments}

This work was supported by project 13-11473S of Grant Agency of the Czech Republic. I.P.R. acknowledges support by the Russian Foundation for Basic Research (project 14-02-90438_Ucr_a) and NASc of Ukraine (project 07-02-14).

\section{References}

[1] V.V. Laguta, M.D. Glinchuk, M. Maryško, R.O. Kuzian, S.A. Prosandeev, S.I. Raevskaya, V.G. Smotrakov, V.V. Eremkin, I.P. Raevski, Phys. Rev. B 87, 064403 (2013).

[2] R.O. Kuzian, I.V. Kondakova, A.M. Daré, V.V. Laguta, Phys. Rev. B 89, 024402 (2014).

[3] V. Bonny, M. Bonin, P. Sciau, K.J. Schenk, G. Chapuis, Solid State Commun. 102, 347 (1997).

[4] B. Fraygola, N. Frizon, M. Lente, A. Coelho, D. Garcia, J. Eiras, Acta Mater. 61, 1518 (2013).

[5] V. Laguta, J. Rosa, L. Jastrabik, R. Blinc, P. Cevc, B. Zalar, M. Remskar, S. Raevskaya, I. Raevski, Mater. Res. Bull. 45, 1720 (2010). 